\title{
Trauma exposure and alcohol use disorder among prisoners in Jimma Zone correctional institution, Southwest Ethiopia: a cross-sectional study
}

\author{
Yimenu Yitayih ${ }^{1 *}$, Matiwos Soboka ${ }^{1}$, Elias Tesfaye ${ }^{1}$, Mubarek Abera ${ }^{1}$, Almaz Mamaru ${ }^{1}$ and Kristina Adorjan ${ }^{2,3,4}$
}

\begin{abstract}
Objective: Trauma exposure and alcohol use are closely related, and large proportion of trauma-exposed individuals use alcohol. The data presented in this paper were obtained as part of a study on substance use disorder and associated factors among prisoners in the correctional institution in Jimma, Southwest Ethiopia. Therefore, in this study we examined comorbidity of traumatic life experiences and alcohol use disorder in inmates of correctional institution in Jimma, Southwest Ethiopia.

Results: The overall prevalence of lifetime alcohol use disorder was $40.1 \%$, and the prevalence of alcohol use disorder among prisoners with lifetime trauma exposure was 44.0\%. Participants with multiple trauma exposures had 2.5-fold higher odds of association for alcohol use disorder than their counterparts ( $A O R=2.47$ [1.23-4.94]). Living in urban areas (AOR $=4.86$ [2.38-9.94]), presence of psychopathy ( $A O R=3.33[1.25-8.86])$, khat abuse (AOR $=7.39[3.99-$ 13.68]), and nicotine dependence ( $A O R=2.49$ [1.16-5.34]) were also positively associated with alcohol use disorder. The prevalence of alcohol use disorder was higher among prisoners with lifetime trauma exposure. Also, this study indicates that prisoners with multiple trauma exposures had higher odds of association for alcohol use disorder than those with no trauma exposure. A public health intervention targeting survivors of traumatic experiences needs to be designed and implemented.
\end{abstract}

Keywords: Crime, Prisoners, Trauma, Alcohol use disorder, Khat abuse, Tobacco dependence

\section{Introduction}

Traumatic events are extremely prevalent; they are related to adverse mental health and have social and economic consequences [1]. The prevalence of exposure to traumatic events according to DSM-5 criteria is $89.7 \%$ [2]. A national trauma comorbidity report found that $60 \%$ of men and $50 \%$ of women experience trauma in their lifetime [3]. Such events are an almost universal experience among prisoners [1]. One study found trauma exposure rates ranging from 62.4 to $87.0 \%$ among incarcerated adult males [4]. Another study showed that $22 \%$ to $43 \%$

\footnotetext{
*Correspondence: yemenu2007@gmail.com

${ }^{1}$ Department of Psychiatry, Faculty of Medical Sciences, Jimma University, Jimma, Ethiopia

Full list of author information is available at the end of the article
}

of patients with posttraumatic stress disorder (PTSD) develop a substance use disorder over the course of their lives [5]. Recent research has also identified PTSD to be a risk factor for substance use relapse and worse treatment outcome [6,7].

Studies have identified several potential mechanisms to explain the relationship between trauma exposure and AUDs. For example, Boys and Marsden defined the self-medication hypothesis as a specific type of functional substance use [8]. In the context of substance use, the self-medication hypothesis suggests that people use alcohol to suppress or reduce the burden of posttraumatic symptoms [9]. In addition, alcoholic patients with a high trauma load often functionally consume alcohol to cope with sleep issues, depression, or intrusive memories [10]. According to the behavioral sensitization paradigm, 
repeated administration of substances or exposure to stress can cause sensitization of dopamine neurons, resulting in a higher dopamine release in response to subsequent stress and substance abuse [11].

The high-risk hypothesis claims that people with a substance disorder have a higher likelihood of developing PTSD because their risky lifestyle is likely to lead to adverse, stressful, and negative life experiences, which can then lead to PTSD $[12,13]$. The vulnerability may be influenced by a lack of coping strategies or neurochemical brain changes caused by substance use [14].

The data presented in this paper were obtained as part of a study on substance use disorder and associated factors among prisoners in the correctional institution in Jimma, Southwest Ethiopia [15]. As noted, previous research has addressed the association between trauma exposure and substance use disorders in general [15]; however, to our knowledge the potential association of traumatic life events with problematic alcohol use has not been examined among prison populations in Ethiopia. Therefore, this study aimed to assess trauma exposure and AUD in prisoners in a correctional institution in Jimma, Southwest Ethiopia.

\section{Main text}

Method and materials

\section{Study design and setting}

We conducted a cross-sectional study in the correctional institution in Jimma, Southwest Ethiopia over a 4-week period (June-July) in 2017. In total, the facility houses 1460 prisoners (1418 men and 42 women).

\section{Sample size}

The sample size (n) was calculated by using the single population proportion formula, $(\mathrm{n}=z 2 p q / d 2)$ and assuming a prevalence $(\mathrm{P})$ of $50 \%$, i.e. 0.5 (because no similar study has been performed among a prison population in Ethiopia), a 95\% confidence interval (CI) $(Z \alpha / 2=1.96)$, a $5 \%$ margin of error $(D, 0.05)$, and a nonresponse rate of $10 \%$. Thus, the final sample size required was 336.

\section{Study procedures}

We used a systematic random sampling technique to select study participants. A total of 1460 prisoners were eligible for the study. The sampling interval was $1460 / 336=4$. Data were collected by five Master of Science in Psychiatry students. All data collectors were given training on the study. The five students were supervised by two Masters of Science in Public Health students and the principal investigator. A pre-test was conducted on $5 \%$ of the sample in the Agaro prison, which is located $45 \mathrm{~km}$ away from Jimma; on the basis of the responses in the pre-test, we corrected some ambiguous words on the data collection questionnaire. We assessed the presence of AUDs with the Alcohol Use Disorders Identification Test (AUDIT) which was developed by the World Health Organization (26). An AUDIT score $\geq 8$ is considered to indicate an AUD. The sensitivity and specificity of AUDIT for AUD are 0.90 and 0.80 respectively [16] and the reliability of AUDIT in this study was 0.87 (Cronbach's $\alpha$ ). Adverse traumatic life events were assessed with the Life Events Checklist (LEC) (positive score if at least one traumatic event was recorded). The LEC was developed by the National Center for Posttraumatic Stress Disorder to aid in the detection of PTSD; it has been widely used in cross-cultural settings and is predictive of AUD, anxiety, depression, and PTSD [17]. We assessed nicotine dependence by the Fagerstrom Test for Nicotine Dependence (FTND) in which a score $\geq 1$ indicates nicotine dependence [18]. The reliability of the FTND in this study was 0.80 (Cronbach's $\alpha$ ). To evaluate khat abuse we used the Drug Abuse Screening Test (DAST) a score $\geq 3$ indicates abuse [19]. The reliability of the DAST in this study was 0.88 (Cronbach's $\alpha$ ). In addition, we used a questionnaire to assess the following variables for AUD: socioeconomic factors (age, sex, marital status, ethnicity, religion, educational status, occupation, income); environmental factors and behavioral and mental health factors. We assessed social support with the Oslo 3-item Social Support Scale [20] and psychopathy with the Psychopathy Checklist: Screening Version (PCL: SV; cutoff score $\geq 13$ ) [21]; the sensitivity of the PCL-SV was 0.94 , the specificity, 0.85 , and the reliability in this study, 0.86 (Cronbach's $\alpha$ ).

\section{Data processing and analysis}

After the tests and questionnaires had been checked for completeness, EpiData Version 3.1 was used to enter the data. Then, the data were exported to the Statistical Package for Social Science version 21.0 for further analysis, and binary logistic regression analysis was used for both bivariate and multivariate analysis to explore associations and identify variables independently associated with AUD. Factors associated with the outcome variables that had a $P$ value $<0.25$ in the bivariate analysis were included in the multivariable analysis. Statistical significance was set at $\mathrm{P}<0.05$.

\section{Results}

\section{Socio-demographic characteristics}

A total of 329 prisoners participated in the study. The response rate was $97.9 \% ; 2.3 \%(n=7)$ did not participate because they were unwilling to be interviewed about their substance use histories. Detailed information on socio-demographic characteristics is provided in Table 1. 
Table 1 Socio-demographic characteristics of prisoners $(n=329)$ in the correctional institution in Jimma, Southwest Ethiopia

\begin{tabular}{lrrl}
\hline Continuous variables & Median & MAD & Range \\
\hline Age (years) & 26 & 8.9 & $16-86$ \\
Income [Birr/months] & 1000 & 1112.0 & $0-35,814$ \\
Time in prison (months) & 9 & 10.4 & $1-120$ \\
$\begin{array}{l}\text { Duration of sentence } \\
\text { (months) }\end{array}$ & 39 & 46.0 & $0-360$ \\
\hline
\end{tabular}

\begin{tabular}{|c|c|c|}
\hline Categorical variables & $\mathbf{n}$ & Percent \\
\hline \multicolumn{3}{|l|}{ Sex } \\
\hline Male & 307 & 93.3 \\
\hline Female & 22 & 6.7 \\
\hline \multicolumn{3}{|l|}{ Place of residence } \\
\hline Urban & 223 & 67.8 \\
\hline Rural & 106 & 32.2 \\
\hline \multicolumn{3}{|l|}{ Religion } \\
\hline Muslim & 181 & 55.0 \\
\hline Orthodox & 97 & 29.5 \\
\hline Protestant & 38 & 11.6 \\
\hline Catholic & 13 & 4.0 \\
\hline \multicolumn{3}{|l|}{ Marital status } \\
\hline Single & 175 & 53.2 \\
\hline Married & 124 & 37.7 \\
\hline Widowed & 23 & 7.0 \\
\hline Divorced/separated & 7 & 2.1 \\
\hline \multicolumn{3}{|l|}{ Level of education } \\
\hline Primary education & 178 & 54.1 \\
\hline Secondary education & 94 & 28.6 \\
\hline College and above & 30 & 9.1 \\
\hline No formal education & 27 & 8.2 \\
\hline \multicolumn{3}{|l|}{ Occupation } \\
\hline Employed & 134 & 40.7 \\
\hline Farmer & 99 & 30.1 \\
\hline Student & 43 & 13.1 \\
\hline Unemployed & 36 & 10.9 \\
\hline $\begin{array}{l}\text { Other } \\
\text { (housewife/dressmaker) }\end{array}$ & 17 & 5.2 \\
\hline
\end{tabular}

The median age of the participants was 26 years (median absolute deviation [MAD] 8.9). The majority of the participants had been residing in urban areas before imprisonment and were unmarried. The most common religion was Muslim (see Table 1).

\section{Prevalence of lifetime trauma exposure and alcohol use disorder}

Of the 329 participants, 209 (63.5\%) had lifetime trauma exposure and 132 (40.1\%) had AUD. Of the 209 prisoners with lifetime trauma exposure, 92 (44.0\%) had AUD in the 12 months before imprisonment: 44 (21.1\%) had hazardous drinking, 16 (7.7\%) had harmful drinking, and 32 (15.8\%) had alcohol dependence; 117 (56\%) had no alcohol use problem. The most commonly encountered types of trauma in the whole group and in the prisoners with AUD were transportation accidents (whole group: $\mathrm{n}=126,38.3 \%$; prisoners with AUD: $\mathrm{n}=69,54.8 \%)$ and physical assault (whole group: $\mathrm{n}=91,27.7 \%$; prisoners with AUD: $n=64,70.3 \%$ ) (see Table 2). In the whole group, 146 (44.4\%) participants had experienced two and more traumatic events. Among the prisoners with AUD $(\mathrm{n}=119), 27(22.7 \%)$ had no exposure to a traumatic life event, 23 (19.3\%) had experienced one traumatic life event, and 69 (58\%) had experienced multiple traumatic life events.

\section{Factors associated with alcohol use disorder}

Multivariable logistic regression showed that experiencing multiple traumatic life events was significantly associated with AUD: Prisoners who had experienced multiple traumatic life events were almost three times more likely to develop AUD than prisoners with no exposure to a traumatic life event (adjusted odds ratio 2.47, 95\% CI $1.23,4.94)$ (see Table 3).

\section{Discussion}

The present study examined the presence of trauma exposure and AUD among prisoners in a correctional institution in Jimma, Southwest Ethiopia. We found that prisoners with trauma exposure were at greater risk for AUD than prisoners with no trauma exposure: 92 (44.0\%) of the prisoners with lifetime trauma exposure had an AUD in the 12 months before imprisonment, compared with $27(22.5 \%)$ of the prisoners without lifetime trauma exposure.

Participants who had been exposed to multiple traumatic life events had higher odds of association for AUD than participants with no exposure to traumatic life events. This finding is in agreement with a study performed in Israel, which found that exposure to traumatic events was a factor for alcohol use [22]. People exposed to multiple traumatic life events are hypothesized to use alcohol to cope with negative emotional feelings, such as anger, despair, and emptiness, and to numb themselves from the experience of the traumatic event [23]. People might also use alcohol as a coping strategy to reduce awareness of the traumatic event [23].

Previous research has also provided substantial support for the self-medication hypothesis in the context of alcohol use in PTSD. Boys and Marsden found that drugs are intentionally used to suppress, control or inhibit negative affective states [8]. Furthermore, Jacobsen et al. [5] found evidence of the intentional use of alcohol to suppress typical PTSD symptoms in 
Table 2 Lifetime trauma exposure among prisoners $(n=329)$ in the correctional institution in Jimma, Southwest Ethiopia

\begin{tabular}{lll}
\hline Variables & \multicolumn{2}{l}{ Alcohol use disorder } \\
\cline { 2 - 3 } & No, $\mathrm{n}(\%)$ & Yes, $\mathrm{n}(\%)$ \\
\hline
\end{tabular}

Transportation accident (for example, car accident, boat accident, train wreck, plane crash)

$\begin{array}{lll}\text { Yes } & 57(45.2) & 69(54.8)\end{array}$

No $153(75.4) \quad 50(24.6)$

Physical assault (for example, being attacked, hit, slapped, kicked, beaten up)

$\begin{array}{lrl}\text { Yes } & 27(29.7) & 64(70.3) \\ \text { No } & 183(76.9) & 55(23.1)\end{array}$

Assault with a weapon (for example, being shot, stabbed, threatened with a knife, gun, or bomb)

$\begin{array}{lcc}\text { Yes } & 32(42.1) & 44(57.9) \\ \text { No } & 178(70.4) & 75(29.6) \\ \text { Fire or explosion } & & \\ \text { Yes } & 21(33.9) & 41(66.1) \\ \text { No } & 189(70.8) & 78(29.2) \\ \text { Sudden, unexpected death of someone close to you } & \\ \text { Yes } & 18(34.0) & 35(66.0) \\ \text { No } & 192(69.6) & 84(30.4)\end{array}$

Sexual assault (rape, attempted rape, made to perform any type of sexual act through force or threat of harm)

$\begin{array}{lrr}\text { Yes } & 6(15.0) & 34(85.0) \\ \text { No } & 204(70.6) & 85(29.4)\end{array}$

Natural disaster (for example, flood, hurricane, tornado, earthquake)

$\begin{array}{lrr}\text { Yes } & 7(17.9) & 32(82.1) \\ \text { No } & 203(70.0) & 87(30.0) \\ \begin{array}{l}\text { Severe human suffering } \\ \text { Yes }\end{array} & \\ \text { No } & 15(35.7) & 27(64.3) \\ & 195(67.9) & 92(32.1)\end{array}$

Sudden, violent death (for example, homicide, suicide)

$\begin{array}{lrr}\text { Yes } & 12(31.6) & 26(68.4) \\ \text { No } & 198(68.0) & 93(32.0) \\ \text { Life-threatening illness or injury } & \\ \text { Yes } & 16(43.2) & 21(56.8) \\ \text { No } & 194(66.4) & 98(33.6)\end{array}$

Other unwanted or uncomfortable sexual experience

$\begin{array}{lrl}\text { Yes } & 3(13.0) & 20(87.0) \\ \text { No } & 207(67.6) & 99(32.4)\end{array}$

Serious injury, harm, or death you caused to someone else

$\begin{array}{lrr}\text { Yes } & 12(40.0) & 18(60.0) \\ \text { No } & 198(66.2) & 101(33.8)\end{array}$

Combat or exposure to a war zone (in the military or as a civilian)

$\begin{array}{lrr}\text { Yes } & 12(42.9) & 16(57.1) \\ \text { No } & 198(65.8) & 103(34.2)\end{array}$

Captivity (for example, being kidnapped, abducted, held hostage, prisoner of war)

$\begin{array}{rrr}\text { Yes } & 8(44.4) & 10(55.6) \\ \text { No } & 202(65.0) & 109(35.0)\end{array}$

Table 2 (continued)

\begin{tabular}{|c|c|c|}
\hline \multirow[t]{2}{*}{ Variables } & \multicolumn{2}{|c|}{ Alcohol use disorder } \\
\hline & No, $n(\%)$ & Yes, n (\%) \\
\hline \multicolumn{3}{|c|}{ Serious accident at work, home, or during recreational activity } \\
\hline Yes & $14(60.9)$ & $9(39.1)$ \\
\hline No & $196(64.1)$ & $110(35.9)$ \\
\hline \multicolumn{3}{|c|}{$\begin{array}{l}\text { Exposure to toxic substance (for example, dangerous chemicals, radia } \\
\text { tion) }\end{array}$} \\
\hline Yes & $4(36.4)$ & $7(63.6)$ \\
\hline No & $206(64.8)$ & $112(35.2)$ \\
\hline \multicolumn{3}{|c|}{ Any other very stressful event or experience } \\
\hline Yes & $6(25.0)$ & $18(75.0)$ \\
\hline No & $204(66.9)$ & $101(33.1)$ \\
\hline
\end{tabular}

particular. Additionally, neuropharmacological studies have provided clear evidence that alcohol is effective in self-medicating PTSD and that withdrawal from alcohol can reactivate the symptoms of PTSD $(5,6$, and 9). Waldrop et al. [7] showed that use of alcohol can help PTSD patients cope with sleep issues, depression, or intrusive memories and reduce physical hyperarousal. In the present study, the group of people who consumed alcohol was also the group with the highest trauma load. Thus, we can assume that the prevalence of PTSD was very high among these respondents and that they intentionally used substances to better deal with symptoms from the PTSD spectrum.

Exposure to traumatic events was higher among prisoners in the Jimma correctional institution, with almost two-thirds reporting having experienced a traumatic event. Although multiple exposures to traumatic events was a significant risk factor for alcohol use among prisoners, the relationship between multiple exposures to traumatic events and alcohol use is more nuanced. Although we cannot establish causality from our data, mental health professionals should recognize that multiple traumatic exposures among prisoners may be a particular risk factor for AUD. These findings also underscore the need for mental health professionals who work with prisoners who have been exposed to a variety of traumatic experiences to thoroughly assess the prisoners for AUD. Given the many barriers to help-seeking in this population, these findings suggest that treatment should target multiple psychopathologies at once. A primary health physician is thus a critical link to substance use treatment or treatment for trauma exposure for prisoners. Further research on integrated models of treatment should examine the effectiveness of such models in addressing trauma exposure and AUD among prisoners. 
Table 3 Multivariable logistic regression analysis for independent predictors of alcohol use disorder among prisoners $(\mathbf{n}=329)$ in a correctional institution in Jimma, Southwest Ethiopia

\begin{tabular}{|c|c|c|c|}
\hline \multirow[t]{2}{*}{ Variable } & \multicolumn{2}{|c|}{ Alcohol use disorder } & \multirow[t]{2}{*}{ AOR $(95 \% \mathrm{Cl})$} \\
\hline & No (\%) & Yes (\%) & \\
\hline \multicolumn{4}{|l|}{ Psychopathy } \\
\hline No & $200(69.4)$ & $88(30.6)$ & Reference value \\
\hline Yes & $10(24.4)$ & $31(75.6)$ & $3.33(1.25-8.86)$ \\
\hline \multicolumn{4}{|l|}{ Adverse traumatic life event } \\
\hline No exposure to traumatic life event & $93(77.5)$ & $27(22.5)$ & Reference value \\
\hline Exposure to one traumatic life event & $40(63.5)$ & $23(36.5)$ & $2.04(0.86-4.88)$ \\
\hline Exposure to multiple traumatic life events & $77(52.7)$ & $69(47.3)$ & $2.47(1.23-4.94)$ \\
\hline \multicolumn{4}{|l|}{ Place of residence } \\
\hline Rural & $89(84.0)$ & $17(16.0)$ & Reference value \\
\hline Urban & $121(54.3)$ & $102(45.7)$ & $4.86(2.38-9.94)$ \\
\hline \multicolumn{4}{|l|}{ Khat abuse } \\
\hline No & $159(83.2)$ & $32(16.8)$ & Reference value \\
\hline Yes & $51(37.0)$ & $87(63.0)$ & $7.39(3.99-13.68)$ \\
\hline \multicolumn{4}{|l|}{ Nicotine dependence } \\
\hline No & $186(70.5)$ & $78(29.5)$ & Reference value \\
\hline Yes & $24(36.9)$ & $41(63.1)$ & $2.49(1.16-5.34)$ \\
\hline
\end{tabular}

AOR adjusted odds ratio, $95 \% \mathrm{Cl} 95 \%$ confidence interval

Reference value: In the analysis, this variable indicated a lower likelihood of alcohol use disorder; it was coded as zero in SPSS logistic regression

In summary, this study has a significant clinical impact in that it will help prison health services meet the complex mental health needs of prisoners. It demonstrates the relevance of integrated and socially acceptable care for treatment of trauma and AUDs in prisons.

\section{Conclusion}

This study in an Ethiopian prison found a high prevalence of AUD among prisoners with lifetime trauma exposure. Prisoners with greater trauma exposure had higher odds of association for AUD than those with no trauma exposure. This study highlights that a public health intervention targeting survivors of traumatic experience needs to be designed and implemented to decrease the risk of AUD and that there is a critical need to provide treatment for prisoners with AUD.

\section{Limitations}

- AUD were based on self-reports that the prisoners provided during an interview and therefore the prevalence of AUD may have been underestimated.

- Participants may have under reported or denied alcohol use because of social desirability bias.

- AUDIT has not yet been validated in prison populations.
- Data on mental illness were also based on selfreports, so they may be biased, and we could not verify the validity of the participants' responses through a psychiatric interview.

\begin{abstract}
Abbreviations
AOR: adjusted odds ratio; AUD: alcohol use disorder; AUDIT: alcohol use disorders identification test; COR: crude odds ratio; DAST: drug abuse screening test; FTND: Fagerstrom Test for Nicotine Dependence; MAD: median absolute deviation.
\end{abstract}

\section{Acknowledgements}

We would like to thank Jimma University for granting ethical approval. Our deep thanks go to all study participants who spent their valuable time responding to the questions in this study. We thank also Jacquie Klesing, Board-certified Editor in the Life Sciences (ELS), for editing assistance with the manuscript.

\section{Authors' contributions}

YY wrote the protocol, participated in data collection, analyzed the data and wrote the manuscript. MS, ET, MA, AM, and KA made substantial contributions to revising and approving the protocol, analyzing the data, and revising subsequent drafts of the manuscript. All authors read and approved the final manuscript.

Funding

No funding was received for this study.

\section{Availability of data and materials}

The datasets generated and analyzed during the current study are part of an ongoing project, and we will make them available to organizations and individuals upon official request. 


\section{Ethics approval and consent to participate}

The study protocol was approved by the Research Ethics and Approval Committee of the Jimma University Institute of Health (IHRERB/755/2017) Participation in the study was voluntary. If participants decided not to participate, there were no negative consequences. Participants could withdraw from the study at any time without giving a reason and without any negative consequences. Verbal consent was obtained from the prisoners because some of them were illiterate or unfamiliar with the use of forms and to protect the anonymity of participants. The Research Ethics and Approval Committee of the Jimma University Institute of Health allowed us to accept verbal consent from study participants. Even though the information was not provided in paper form, we provided participants with all the necessary information in a verbal consent procedure before obtaining their consent. We explained to the participants that selection for participation in the study was random and that they had the right not to respond to questions that they were not comfortable with and also had the right to ask questions. After data entry was complete, the questionnaires were kept securely locked away. Study participants who did not want to participate in the study but had been exposed to trauma and had AUD were referred to the Jimma University Medical Centre.

\section{Consent for publication}

Not applicable.

\section{Competing interests}

The authors declare that they have no competing interests.

\section{Author details}

${ }^{1}$ Department of Psychiatry, Faculty of Medical Sciences, Jimma University, Jimma, Ethiopia. ${ }^{2}$ Institute of Psychiatric Phenomics and Genomics (IPPG), Medical Center of the University of Munich, Munich, Germany. ${ }^{3}$ Departments of Psychiatry and Psychotherapy, Medical Center of the University of Munich, Munich, Germany. ${ }^{4}$ Center for International Health, Ludwig-MaximiliansUniversität, Munich, Germany.

Received: 6 November 2019 Accepted: 11 November 2019

Published online: 19 November 2019

\section{References}

1. Carlson BE, Shafer MS. Traumatic histories and stressful life events of incarcerated parents: childhood and adult trauma histories. Prison J. 2010;90(4):475-93.

2. Kilpatrick DG, Resnick HS, Milanak ME, Miller MW, Keyes KM, Friedman MJ. National estimates of exposure to traumatic events and PTSD prevalence using DSM-IV and DSM-5 criteria. J Trauma Stress. 2013;26(5):537-47.

3. Sadock BJ, Sadock VA, Ruiz P. Kaplan and Sadock's synopsis of psychiatry: behavioral sciences/clinical psychiatry. 11th ed. Philadelphia: Wolters Kluwer; 2014.

4. Gibson LE, Holt JC, Fondacaro KM, Tang TS, Powell TA, Turbitt EL. An examination of antecedent traumas and psychiatric comorbidity among male inmates with PTSD. J Trauma Stress. 1999;12(3):473-84.

5. Jacobsen LK, Southwick SM, Kosten TR. Substance use disorders in patients with posttraumatic stress disorder: a review of the literature. Am J Psychiatry. 2001;158(8):1184-90.

6. Odenwald M, Semrau P. Reducing dropout among traumatized alcohol patients in detoxification treatment: a pilot intervention study. Eur Addict Res. 2012;18(2):54-63.
7. Waldrop AE, Back SE, Verduin ML, Brady KT. Triggers for cocaine and alcohol use in the presence and absence of posttraumatic stress disorder. Addict Behav. 2007:32(3):634-9.

8. Boys A, Marsden J, Stillwell G, Hatchings K, Griffiths P, Farrell M. Minimizing respondent attrition in longitudinal research: practical implications from a cohort study of adolescent drinking. J Adolesc. 2003;26(3):363-73.

9. Khantzian EJ, Treece C. DSM-III psychiatric diagnosis of narcotic addicts: recent findings. Arch Gen Psychiatry. 1985;42(11):1067-71.

10. Kaysen D, Dillworth TM, Simpson T, Waldrop A, Larimer ME, Resick PA Domestic violence and alcohol use: trauma-related symptoms and motives for drinking. Addict Behav. 2007;32(6):1272-83.

11. Adorjan K, Odenwald M, Widmann M, Tesfaye M, Tessema F, Toennes S, Suleman S, Papiol S, Soboka M, Mekonnen Z, Rockstroh B. Khat use and occurrence of psychotic symptoms in the general male population in Southwestern Ethiopia: evidence for sensitization by traumatic experiences. World Psychiatry. 2017;16(3):323.

12. Cottler LB, Compton WM, Mager D, Spitznagel EL, Janca A. Posttraumatic stress disorder among substance users from the general population. Am J Psychiatry. 1992;149(5):664-70.

13. Potthast N, Catani C. Trauma und Sucht: implikationen für die Psychotherapie. Sucht. 2012;58(4):227-35.

14. Brown PJ, Wolfe J. Substance abuse and post-traumatic stress disorder comorbidity. Drug Alcohol Depend. 1994;35(1):51-9.

15. Yitayih Y, Abera M, Tesfaye E, Mamaru A, Soboka M, Adorjan K. Substance use disorder and associated factors among prisoners in a correctional institution in Jimma, Southwest Ethiopia: a cross-sectional study. BMC Psychiatry. 2018:18(1):314.

16. Babor TF, Higgins-Biddle JC, Saunders JB, Monteiro MG. AUDIT. The alcohol use disorders identification test. Guidelines for use in primary care. 2nd ed. Geneva: World Health Organization; 2001.

17. Gray MJ, Litz BT, Hsu JL, Lombardo TW. Psychometric properties of the life events checklist. Assessment. 2004;11(4):330-41.

18. Heatherton TF, Kozlowski LT, Frecker RC, Fagerström KO. The Fagerström test for nicotine dependence: a revision of the Fagerström Tolerance Questionnaire. Br J Addict. 1991;86:1119-27.

19. Wazema DH, Madhavi K. Prevalence of Khat abuse and associated factors among undergraduate students of Jimma University, Ethiopia. Int J Res Med Sci. 2017:3:1751-7.

20. Bøen H, Dalgard OS, Bjertness E. The importance of social support in the associations between psychological distress and somatic health problems and socioeconomic factors among older adults living at home: a cross sectional study. BMC Geriatr. 2012:12:27.

21. Hart SD, Cox DN, Hare RD. Manual for the hare psychopathy checklist: screening version (PCL: SV). Toronto: Multi-Heath Systems; 1995.

22. Walsh K, Elliott JC, Shmulewitz D, Aharonovich E, Strous R, Frisch A, et al. Trauma exposure, posttraumatic stress disorder and risk for alcohol, nicotine, and marijuana dependence in Israel. Compr Psychiatry. 2014;55(3):621-30.

23. Khantzian EJ. The self-medication hypothesis of substance use disorders: a reconsideration and recent applications. Harv Rev Psychiatry. 1997:4(5):231-44.

\section{Publisher's Note}

Springer Nature remains neutral with regard to jurisdictional claims in published maps and institutional affiliations. 